\title{
POSTSYNAPTIC SIMULATOR: AN OPEN-SOURCE VISUAL INTERACTIVE SIMULATION FOR TEACHING ACTION POTENTIALS
}

\author{
Alexander Salgo ${ }^{1}$, Galen O'Shea ${ }^{2}$, Kim Hellemans ${ }^{3,4}$ and Jim Davies ${ }^{2,4}$ \\ ${ }^{1}$ Queen's University, School of Computing, Canada \\ ${ }^{2}$ Carleton University, Dept. of Cognitive Science, Canada \\ ${ }^{3}$ Carleton University, Dept. of Neuroscience, Canada \\ ${ }^{4} \mathrm{Dr}$.
}

\begin{abstract}
We introduce an open-source game-like educational software that teaches users about how action potentials work. The Postsynaptic Simulator requires users to demonstrate understanding of neural mechanisms to progress through levels, each of which teaches a different aspect of neuron activity.
\end{abstract}

KEYWORDS

Edutainment, Education, Neuroscience, Serious Games, Action Potentials

\section{INTRODUCTION}

Neuroscience undergraduates have a relatively hard time understanding the chain of events leading up to neuron firing, known in neuroscience as an "action potential." In introductory textbooks, the role that post-synaptic potentials play in causing an action potential is often unclear. For example, a 2011 textbook reads: "A neuron with thousands of inputs responds no differently from one with only a few inputs. It democratically sums up all inputs that are close together in time and space. The cell-body membrane, therefore, always indicates the summed influences of multiple inputs" (Kolb \& Whishaw, 2011). While descriptions like this are technically correct, they paint a misleading picture of the cell membrane as a homogenously charged surface that sums inputs instantly.

Explanations like these miss out on much of the detail and complexity involved with the process of action potential generation. The rate at which a neuron fires depends on the integration of thousands of inhibitory and excitatory post-synaptic potentials, as well as the shape and electrophysiology of the neuron receiving these potentials (Etherington et al., 2001). People commonly, but mistakenly, believe that any activation at dendrites contributes to charge at the axon hillock, which is the part just before the axon-the charge at this point determines whether the neuron will fire or not. But in truth, the activation has to exceed the decay rate, or the signal will vanish before it reaches the hillock. While a linear explanation of this process is easy for students to digest, it can also cause them to form an incorrect mental model of how electrochemical charge is transferred across the surface of a neuron. Misleading mental models make it difficult for students to learn more complex concepts such as temporal summation, spatial summation, inhibitory post-synaptic potentials, and how individual neurons integrate information.

The mental models that exclude these crucial details are reinforced by current methods of teaching neuroanatomy and electrophysiology. At present, most educators use static diagrams (either from textbooks or drawn manually) when teaching these topics. While diagrams are useful, they fail to capture the dynamic nature of electrophysiology and potentially contribute to misunderstandings. An example of such a diagram is the extremely popular action potential voltage graph, a representation of which is shown in Figure 1: the sharp and near-instantaneous changes in voltage suggest that the action potential is caused by a single large post-synaptic potential. The "failed initiations" shown in the diagram add to this misconception. 
Many educators attempt to supplement this with animated videos that show the process unfolding (Korey, 2009). The nature of video-based education is also limiting, however, and most videos present the same type of linear sequence that the diagrams do.

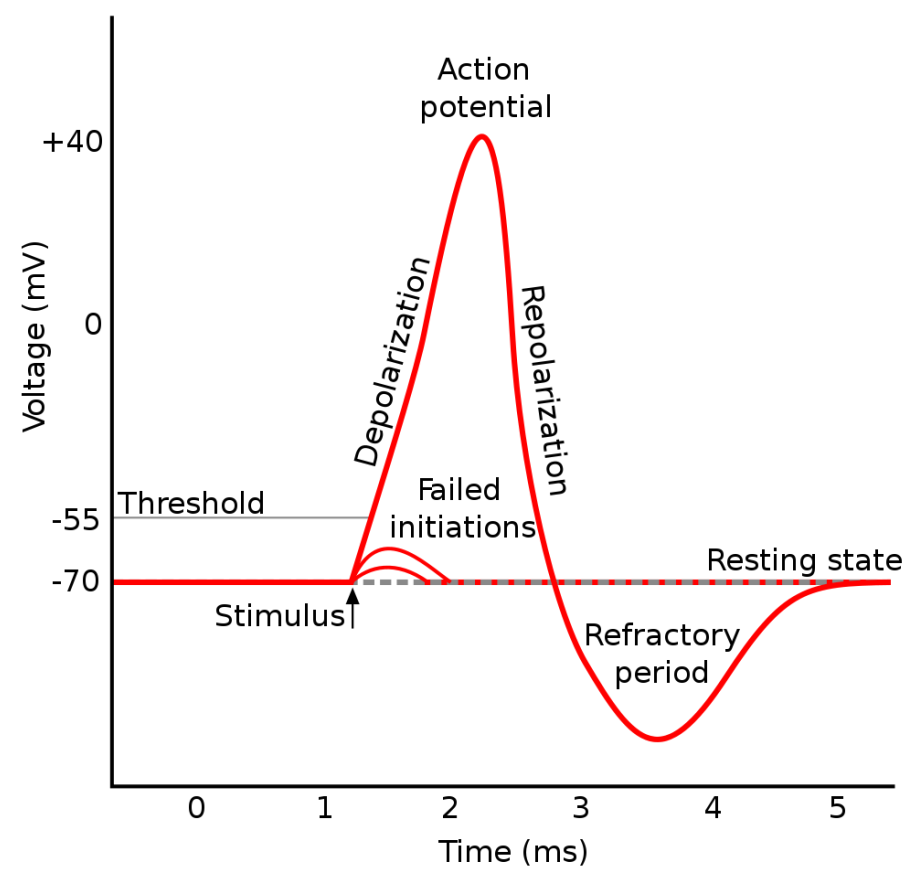

Figure 1. An example commonly used diagram that teaches students about action potentials (tiZom, 2007)

Neither diagrams nor videos allow students to experiment with neural activity in an interactive way. Interactive simulations have been a popular tool for teaching both basic and advanced physics for years (Wieman, Perkins, \& Adams, 2008), and have been shown to aid education in other fields. These simulations are often game-like, with students being given goals to accomplish in order to complete pre-designed scenarios. Stieff and Wilensky (2003) showed that students who used interactive simulations demonstrated a better ability to reason about chemistry problems and relied less on memorized answers. This is indicative of the type of deep understanding that is needed when learning about complex dynamic processes.

It is likely that analogous methods could be employed in neuroscience courses to achieve a similar level of understanding of neural function. In this paper we describe such an interactive learning system for teaching students about how action potentials work.

\subsection{Goals of the System}

The goal of this paper is to present a new interactive simulation program, the Postsynaptic Simulator, that harnesses the benefits of interactivity in a way that has yet to be done in neuroscience. While educational neuroscientific simulations exist (as will be discussed below), they tend to lack some of the core features that make simulation such a useful tool for teaching. The core goals of this new program are as follows:

1. Provide a game-like interactive environment to motivate students to use it (i.e., make it fun).

2. To teach students about basic neuroanatomy, temporal and spatial summation of post-synaptic potentials, and the sequence of events leading up to an action potential in a neuron.

3. To provide an easy-to-customize, open-source framework upon which educators can build additional exercises, with significantly less programming experience than existing programs would require. 
The third goal of this program is perhaps its most unique aspect. This program is designed such that additional levels, complete with customized goals and layouts, can be added using an entry-level programming language. This should also allow for the addition of features that are not present in the base program. The open-source nature of this program will also allow educators to share their levels and add-ons with others, meaning that they can access new supplementary material without having to buy new software.

The way in which these goals will be met is discussed in the section titled Postsynaptic Simulator. First, an overview of some existing programs will help demonstrate why this program is a useful addition to existing computer-based educational tools.

\subsection{Existing Simulations in Neuroscience Education}

This section is an overview (and evaluation) of three simulation programs currently used by educators.

g-PRIME (Lott et al., 2009) is a software tool designed to allow for investigation of various aspects of neurophysiology. It is a multi-purpose program, in that it facilitates both simulation and real data-acquisition. This flexibility makes it useful in lab settings, but the extra functionality is not as useful for early-year undergraduates (who are unlikely to be performing experiments using live neurons). It does not appear to be particularly designed to help teach students how neurons work, but rather how to analyze physiological data.

In terms of simulation, g-PRIME does not directly simulate readings from instruments. Instead, because it allows for both real-time and offline data analysis, educators are able to provide a data file that students can view and analyze using the program. This allows them to perform analyses on data as though they had gathered it themselves. The visual outputs of the program are a series of graphs which correspond to the readings taken. This is useful for teaching students about the types of readings they will encounter in a lab setting but does little to provide an in-depth understanding of why it is that the readings occur.

Because the interface allows for real data analysis, it has a level of complexity that is greater than a program designed purely for increasing conceptual knowledge. This is not a weakness of the program but it makes it difficult to teach new concepts with it. Instead, this program is ideal for students who are already familiar with the basic concepts of neuroanatomy and physiology.

A benefit of this program is that it allows for some customization. By giving students different data to analyze, educators can teach students about diverse neurophysiological phenomena. Unfortunately, this is limited to the those that are easily detected by lab instruments, so fundamental aspects of neuroanatomy are beyond the scope of the program.

Meta-Neuron (Newman \& Newman, 2013) is a simulation designed specifically for educational purposes, and comes with six built-in exercises. These exercises cover similar topics to our program, with simulations that teach students about resting membrane and action potentials. Rather than analysing existing data for a lab exercise (as would g-PRIME), Meta-Neuron generates simulated readings.

Simulating readings allows students to view the responses that they would see if they were stimulating a neuron themselves. The visual outputs of Meta-Neuron are similar to those in g-PRIME, presented in graph-form, with aspects of the process (such as ion levels) presented as an updating line on the graph. While this is useful for students who already understand what is occurring at a molecular level, it does not help students understand the fundamental nature of the electrochemical changes that lead to action potentials.

The simulation environment is also complex. Students interact with the simulation by manipulating parameters that adjust variables involved in the process. This is a complex process for new users and does not intuitively aid students in figuring out the best course of action to achieve their goal.

In terms of customizability, Meta-Neuron is not designed for custom exercises. While educators can give different instructions to students in order to achieve different results, students will always be working with the same interface and the same built-in exercises.

NeuroLab (Schettino, 2014) is designed to teach a variety of neurophysiological concepts to students. It does so in a way that is more conducive to a deep understanding of the phenomena involved. NeuroLab is a set of differently designed simulations. Each of these simulations is short and designed to teach a particular concept such as the temporal integration of inputs or principles of axonal guidance. Simulations are visually straightforward and allow students to explore the physical processes behind neuronal activity. 
Because of the short nature of the exercises, the interface is not overly complicated. Unfortunately, interaction often takes the form of parameter adjustment. This limits users' direct involvement in the on-screen visual simulation. Although this does not stop it from being a useful tool, this is a key aspect that could be improved upon to increase student comprehension.

Neuro-lab is quite flexible: The open-ended nature of simulations allows professors to design their own goals for the students to achieve. Unfortunately, these cannot be built into the program, and as such there are limitations on the complexity of goals that can be assigned. It also may make it difficult for a student to know when they have achieved a desired outcome.

\section{POSTSYNAPTIC SIMULATOR: SYSTEM DESCRIPTION}

Core Functionality: The Postsynaptic Simulator is currently available, free, in the iOS store (for iPhones and iPads). The game is designed to simulate the complex intracellular electrochemical activity that leads to an action potential. It allows students to control the inputs a neuron receives in a more realistic way than those provided by other simulations to date. Rather than showing students graphs representing the ion gradients or electric charge levels at the axon hillock, the Postsynaptic Simulator allows students to see how post-synaptic potentials progress from the synapse to create a graded potential across the membrane.
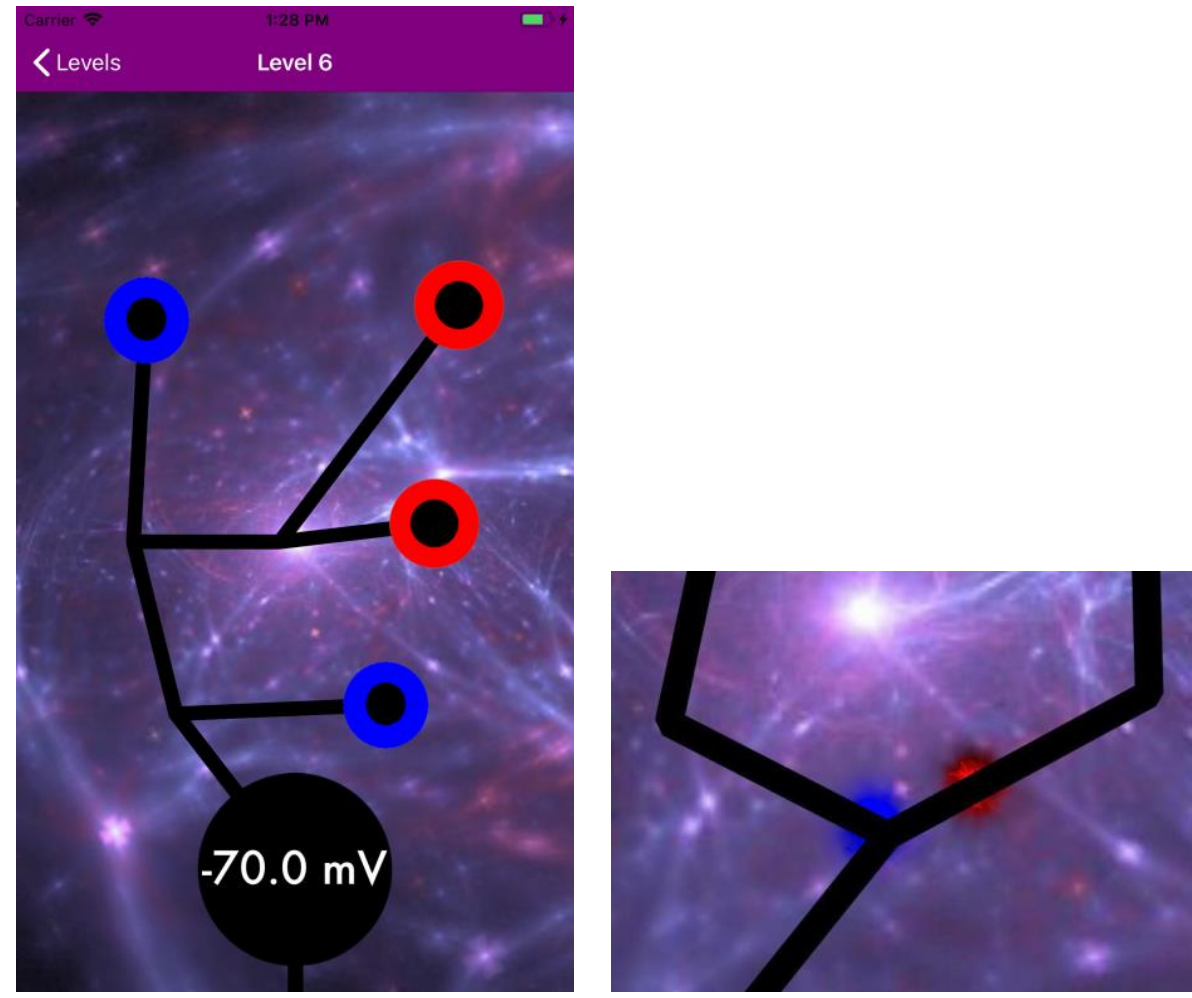

Figure 2. Left: Screenshot of a basic level layout, with dendritic branches (black lines), four synapses (excitatory shown as blue circles and inhibitory shown as red circles), a cell body (black circle with a voltage meter measure in millivolts), and an axon hillock (stemming from the bottom of the cell body). Right: Excitattory and Inhibitory graded potentials that travel toward the hillock (blue and red, respectively)

The graded potential that this program simulates demonstrates the basic principles of electrochemistry in a neuron. It propagates along the cell membrane (that is, the outside of the neuron), degrading with both time and distance; critically, the interface allows for a student to observe how the activation of a synapse causes a degrading wave of electrical activity. This allows students to see why temporal and spatial summation are required for charge to accumulate at the axon hillock. 
Charge gradients within the cell are represented by the colour of cell walls. Red represents more positive charge, blue represents more negative charge, and black represents resting-state charge levels. These colours were chosen because they align with popular depictions of charge. More intense colours (further from black) as well as increased size are used to show the accumulation of charge, which further reinforces the "summation" aspect of neuronal activity.

Interface: The interface of the program has an abstract design, as shown in Figure 2. Students will be able to select a level from the level menu. From there it will take them to the Objective screen, which explains how they can beat the level. Once they press "continue" the game will begin. At any point they can go back to the level menu by clicking the "Levels" button on the top left of the screen. Levels are designed individually in a Level Builder. Each level can have a different layout, different goals, and specific features to design a wide range of levels.

Users interact with levels by touching synapses on the iOS device's touch screen. The synapses are colour coded to whether they are excitatory or inhibitory and are located on dendrites or the cell body (as in the case with real neurons; although a synapse can occur anywhere on the cell membrane, we opted for the two more common regions). A level is won by touching the right combination of synapses at the right times to cause the neuron to fire. Each level is designed like a puzzle, and solving each puzzle teaches something about neuronal activity. That is, they need to understand a concept to be able to progress past the level.

Level Layout: Each level contains one or more neurons, represented by abstract neuron diagrams. These diagrams depict the cell body (as a black circle), one or more dendrites (represented by lines), and an axon (another line extending downwards from the cell body). These diagrams also depict synapses, which are the primary points of interaction that users have with the simulation. Synapses are represented as circles with black central dots. Neuron layouts are customized for each level. Different levels can have different numbers of dendrites located at different points on the cell and can have branches connected in any arrangement.

Levels have "victory conditions." The victory conditions for a level dictate when a student has accomplished the goals of that particular level. These can be simple (e.g., "cause an action potential" or "block an action potential") or they can be extremely complex (e.g., "cause three action potentials in a row without clicking on the same synapse twice.")

Simulation: The simulation of intracellular electrical activity is the core function of this program. The algorithms behind the simulation are designed to strike a balance between realism and clarity. For example, exact simulations of voltage levels are not used, as they would greatly increase the complexity and computational requirements of the program without adding additional clarity. Rough estimates generate depictions on screen similar enough to reality to teach the intended concepts.

Synapses are represented by blue or red circles, identifying excitatory or inhibitory graded potentials, respectively. Dendrites are represented by black paths which extend from the synapses to the cell body. Synapses can be pressed to simulate activation, thereby changing the local membrane potential on the dendrite or cell body. These electrochemical signals (graded potentials) travel along the cell membrane.

The graded potential is represented by red and blue particles that travel from the synapses down through the dendrite. These particles help visualize the nature of graded potentials as they propagate along the cell walls. The charge gradients are represented by the colour blue to signify positive charge from excitatory graded potentials and the colour red to represent negative charge from inhibitory graded potentials.

To help visualize presynaptic spatial summation, each graded potential's size is directly proportional to its strength, with larger glows representing more accumulated charge. There is also a non-linear function, known as synaptic coincidence detection, added into the spatial summation equation. This acts as a multiplier as more charge accumulates. That is, if two excitatory graded potentials meet, they sum to a single, greater graded potential. Inversely to the spatial summation of like charges, the summation of opposite charges is represented by a size decrease, or even disappearance, if the opposite charges are equivalent.

Another key feature is the decay of graded potentials along the path to the cell body. This concept is represented by the graded potential visually fading along with the strength of its charge as it propagates along the dendrite. The decay can be seen in Figure 3.

The cell body is represented by a larger black circle at the bottom of the screen. In it is a voltage meter (see Figure 4), which depicts the state of intracellular activity, and provides feedback to the student's actions in the level. The cell body will begin to glow blue to represent that it is being mostly stimulated by excitatory graded potentials leading to an action potential. In contrast, the cell body will glow red to represent the stimulation of inhibitory graded potentials. The size and intensity of the glow represents the strength to which the cell body is being affected. Stemming from the bottom of the cell body to the bottom of the screen is the 
axon, which is represented by a black line (see the bottom of Figure 2). When the threshold has been achieved, and an action potential is triggered, the axon hillock will fire a wave of charge. Depending on how much the threshold is surpassed, the wave will pulse faster, simulating the way a real action potential would fire (i.e., greater depolarization, increased rate of firing).
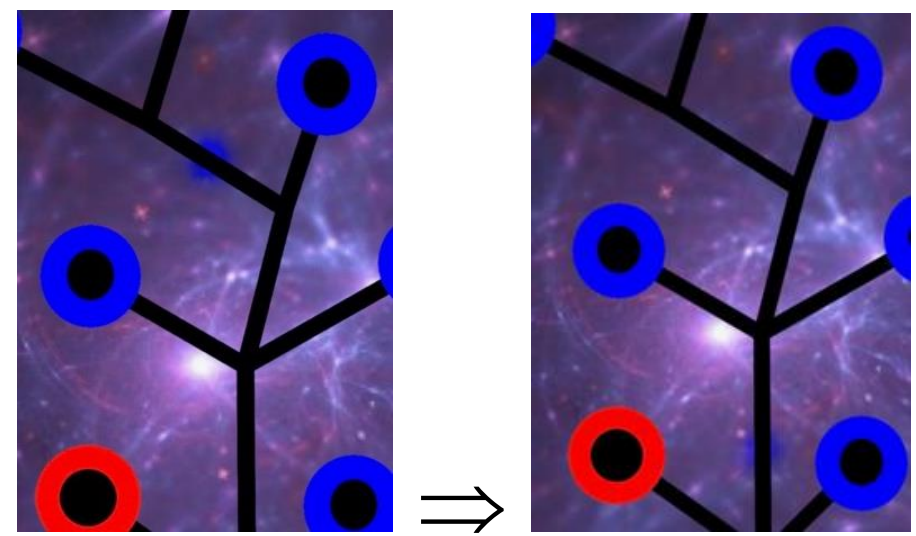

Figure 3. The decay of a graded potentials stength as it propogates through the denfdrite. At time 1, left, the potential (the fuzzy blue glow $1 / 4$ from the top) is brighter and less decayed than at time 2, right (near the bottom)

Customizability: The program currently has several levels, each designed to teach users about some aspect of neuron function. But new levels can be created with a Level Builder, allowing several ways for custom levels to be programmed. As with similar programs, customizing levels or creating new features requires a certain level of programming knowledge. In order to minimize the amount of knowledge needed to create content for this program, we used the swift programming language.

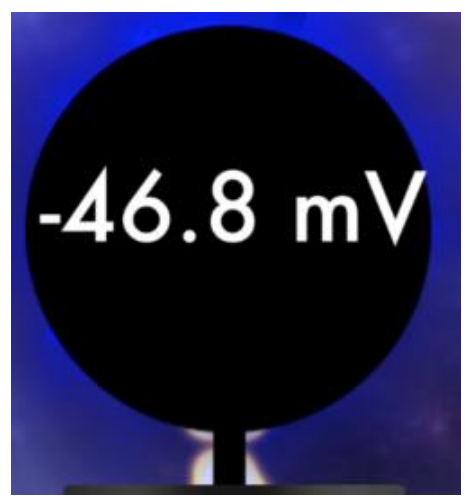

Figure 4. The label indicates the default threshold of $-55 \mathrm{mV}$ has been surpassed. The Cell Body shows a blue halo along the outside, identifying it as being stimulated by excitatory graded potentials. An action potential appears different from a graded potential. It moves in a self-propagating wave along the axon

Swift is a high-level programming language designed to facilitate the creation of mobile applications. Swift is programmed using Xcode which is a free development software built by Apple, Inc. Xcode offers a variety of resources including storyboard functionality (a visual editor) which connects code to the user interface, Spritekit (a game building framework), and a simulator (to test the application on multiple devices). All of these resources, in conjunction with the custom level builder, are beneficial to developers looking to add additional content with little programming experience and minimal lines of code. Swift also increases the application's accessibility as it can also be posted directly to the Apple Appstore. Students will be able to quickly and easily download the app to their mobile devices and access the app wherever they go. They can find the source code and a tutorial to get started at: https://github.com/galenoshea/Postsynaptic-Simulator. The built-in variables that allow for customization are listed in Table 1. 
Table 1. A list of features that can be customized using either a single variable or a new instance of a preprogrammed type of object

\begin{tabular}{lll}
\hline Feature & Variables & Notes \\
\hline Cell Body & Shape & circle and straight lines currently programmed \\
\hline & Size & - \\
\hline & Layout & $\begin{array}{l}\text { Can be connected in any desired format using } \\
\text { connection points }\end{array}$ \\
& Thickness & - \\
\hline Cynapses & Lolour & - \\
\hline & Location & $\begin{array}{l}\text { Can be placed at any point along a charge } \\
\text { surface }\end{array}$ \\
\hline & Size & $\begin{array}{l}\text { Can make synapses easier to click for levels } \\
\text { requiring sequential firing }\end{array}$ \\
\hline & Inhibitory or excitatory & - \\
effect & Strength of effect & - \\
\hline Duration & $\begin{array}{l}\text { How long the synapse remains active for after } \\
\text { activating }\end{array}$ \\
& Count & How many times the synapse can fire.
\end{tabular}

\begin{tabular}{|c|c|c|}
\hline Feature & Variables & Notes \\
\hline \multirow{2}{*}{$\begin{array}{l}\text { Synapses } \\
\text { (cont.) }\end{array}$} & Refractory period & Customizable to any interval. \\
\hline & Automatic firing & $\begin{array}{l}\text { Synapses trigger automatically instead of } \\
\text { requiring user input }\end{array}$ \\
\hline \multirow[t]{3}{*}{ Axons } & Activation threshold & Defaults to $-55 \mathrm{mV}$. customizable \\
\hline & Refractory period & - \\
\hline & Action potential speed & $\begin{array}{l}\text { How fast an action potential moves along the } \\
\text { axon. Proportional to the rate at which the } \\
\text { threshold was surpassed. }\end{array}$ \\
\hline \multirow[t]{2}{*}{$\begin{array}{l}\text { Postsynaptic } \\
\text { Potential }\end{array}$} & Rate of diffusion & $\begin{array}{l}\text { The rate at which charge spreads along } \\
\text { charge surfaces }\end{array}$ \\
\hline & Decay rate & $\begin{array}{l}\text { The rate at which charge gradually returns to } \\
\text { neutral }\end{array}$ \\
\hline \multirow[t]{4}{*}{ Game } & Background colour & $\begin{array}{l}\text { Custom image or colour. Defaults to neuron } \\
\text { background. }\end{array}$ \\
\hline & Window size & Adaptive to all iphones/ipods. \\
\hline & Game timer & Can create custom count down to win/loss \\
\hline & Level objective & Can write custom instructions to play level. \\
\hline
\end{tabular}




\section{CONCLUSION}

The Postsynaptic Simulator provides an excellent framework with which to create supplementary material for introductory neuroscience courses. It provides an intuitive interface for students to explore basic electrophysiology. It allows students to see the ways in which graded potentials propagate and how they can be summed to cause action potentials. Our contribution is the software itself, currently available in the App Store and ready for use in classrooms.

The Postsynaptic Simulator also provides a foundation for further simulations of both individual and connected neurons. By creating a generalizable foundation for this, the program allows for both new and experienced programmers to add features that suit their curriculum. Because it has a Level Builder, new educational modules can be created using it. Instructors who are not comfortable with programming can assign level design as class assignments for groups of students that include some that are comfortable with software.

This program hopes to serve as a valuable addition to introductory neuroscience material, and to help newcomers to the field understand the processes behind causing an action potential. Future work will evaluate the benefits of using the Postsynaptic Simulator, in terms of enjoyment and learning outcomes.

\section{REFERENCES}

Kolb, B., Whishaw, I.Q. and Teskey, G.C., 2011. An introduction to brain and behavior. ( ${ }^{\text {rd }}$ Ed.) New York: Worth.

Korey, C.A., 2009. Teaching undergraduate neuroscience in the digital age. Journal of Undergraduate Neuroscience Education, 8(1), p.A55.

Lott, G.K., Johnson, B.R., Bonow, R.H., Land, B.R. and Hoy, R.R., 2009. g-PRIME: a free, Windows based data acquisition and event analysis software package for physiology in classrooms and research labs. Journal of Undergraduate Neuroscience Education, 8(1), p.A50.

Newman, M.H. and Newman, E.A., 2013. MetaNeuron: a free neuron simulation program for teaching cellular neurophysiology. Journal of Undergraduate Neuroscience Education, 12(1), p.A11.

Reas, C. and Fry, B., 2007. Processing: a programming handbook for visual designers and artists. Cambridge: MA: MIT Press.

Schettino, L.F., 2014. NeuroLab: a set of graphical computer simulations to support neuroscience instruction at the high school and undergraduate level. Journal of Undergraduate Neuroscience Education, 12(2), p.A123.

Stieff, M. and Wilensky, U., 2003. Connected chemistry-incorporating interactive simulations into the chemistry classroom. Journal of Science Education and Technology, 12(3), pp.285-302.

tiZom, O. by en:User:Chris 73 updated by en:User:Diberri converted to SVG by (2007). Schmatic of an action potential without labels. Retrieved from https://commons.wikimedia.org/wiki/File:Action_potential_(no_labels).svg

Wallis, J.D., Anderson, K.C. and Miller, E.K., 2001. Single neurons in prefrontal cortex encode abstract rules. Nature, 411(6840), pp.953-956.

Wieman, C.E., 2006, December. Interactive Simulations for Teaching Physics; What Works, What Doesn't, and Why. In American Astronomical Society Meeting Abstracts (Vol. 209, pp. 249-01). 